\title{
Photoluminescence and deep levels in lattice-matched InGaAsN/GaAs
}

\author{
C. H. Fischer and P. Bhattacharya \\ Solid State Electronics Laboratory, Department of Electrical Engineering and Computer Science, \\ University of Michigan, Ann Arbor, Michigan 48103-2122
}

(Received 3 June 2004; accepted 19 July 2004)

\begin{abstract}
Temperature-dependent photoluminescence measurements and deep level transient spectroscopy have been made to identify defects and deep level traps in InGaAsN alloys lattice-matched to GaAs. The epitaxial layers were grown by molecular beam epitaxy at different substrate temperatures. Defect levels responsible for the quenching of luminescence with increase of temperature have been identified. Several electron trap levels have been identified and characterized in the alloys. The concentrations of all of these increase with $\mathrm{N}$ content in the alloy. A dominant center, with an activation energy of $\mathrm{E}_{\mathrm{T}}=0.44 \mathrm{eV}$, is present in all the samples and its concentration is inversely proportional to photoluminescence intensity. $\mathrm{H}_{2} \mathrm{O}$ and $\mathrm{O}$ present in the source nitrogen are believed to be responsible for the presence of this trap, which also has characteristics similar to the E3 and E4 levels in molecular beam epitaxial GaAs.
\end{abstract}

(C) 2004 American Institute of Physics. [DOI: 10.1063/1.1791757]

\section{INTRODUCTION}

The $\mathrm{Ga}(\mathrm{As}, \mathrm{N})$ dilute nitrides are emerging as important materials for band gap engineering in a host of device applications. ${ }^{1-3}$ These are primarily driven by the uniquely large bowing parameter in the GaAsN system. ${ }^{4}$ However, under near-equilibrium growth conditions, immiscibility, clustering, and the formation of $\mathrm{N}-\mathrm{N}$ pairs and complexes are promoted. The composition range in which GaAs: $\mathrm{N}$ behaves as a regular alloy is very limited. Progress in the epitaxy of these alloys has been largely achieved by growing under farfrom-equilibrium conditions and by using a highly reactive nitrogen precursor as the source. To suppress phase separation effects, the $\mathrm{N}$-containing alloys are usually grown at low temperatures, leading to poor photoluminescence intensity. ${ }^{5}$ This is compensated, to a large extent, by post growth annealing, ${ }^{6,7}$ although the degradation and anneal mechanism are still subjects of study. In spite of these hurdles, lasers containing dilute nitrides in the active region have been demonstrated. $^{8-14}$ The realization of high performance devices serves as a motivation to improve materials quality, including the luminescent and transport properties.

The $\mathrm{Ga}(\mathrm{As}, \mathrm{N})$ alloys grown on GaAs are under biaxial tensile strain. Addition of small amounts of In helps to compensate for this strain and lattice-match the quaternary alloy to the GaAs substrate. At the same time, the addition of indium lowers the band gap slightly. Low-temperature growth under far-from-equilibrium conditions can lead to nonradiative defects and deep levels. Most deep level studies in these materials have been restricted to thin films of the $\mathrm{Ga}(\mathrm{As}, \mathrm{N})$ alloys. In the present study, we have undertaken a detailed characterization of deep levels in InGaAsN grown by molecular beam epitaxy and have correlated these results to temperature-dependent photoluminescence $(\mathrm{PL})$ data from the same set of samples.

\section{EXPERIMENTAL TECHNIQUES}

The epitaxial layers of InGaAsN were grown on (001) Si doped $n^{+}$substrates in a Riber $32 \mathrm{P}$ molecular beam epitaxy
(MBE) system, which is equipped with solid sources for the Group III elements and arsenic and an applied-EPI Uni-Buib radio frequency (RF) plasma source for nitrogen. An ultrahigh-purity blend of nitrogen and argon (14.9 at \% nitrogen) is used with a low flow-conductance aperture to achieve low flow rates of monatomic nitrogen while maintaining sufficient pressure in the plasma source to maintain the plasma. Extremely low gas flow rates of approximately $0.1 \mathrm{sccm}$ are maintained with a needle valve and set to a constant nitrogen partial pressure of $2 \times 10^{-7}$ Torr using a Stanford Research Systems residual gas analyzer on the growth chamber for better consistency between growths. A radiatively coupled thermocouple is used to monitor the temperature of the substrate, which is indium-mounted to a molybdenum block during growth. The temperature offset between the GaAs wafer and the thermocouple is determined by observation of initial oxide desorption from the surface of the wafer by reflective high-energy electron diffraction (RHEED). The offset is assumed constant across the range of growth temperatures and is less than $10^{\circ} \mathrm{C}$ for all samples.

Lattice matching and approximate compositions of the quaternary films were measured by double-axis x-ray diffraction (DXRD) measurements on calibration samples of $0.5 \mu \mathrm{m}$ ternary films (InGaAs and GaAsN). Measurements were taken along the (004) and (115) crystallographic axes to ensure that the films were not relaxed. Once suitable nitrogen plasma-cell parameters and indium cell temperatures were established, these parameters were kept fixed while the gallium flux was varied to produce various lattice-matched compositions. Varying the gallium growth rate from 1.5 to $3 \AA / s$ resulted in nitrogen compositions from $0.55 \%$ to $0.27 \%$, respectively, with negligible impact on the $[\mathrm{In}] /[\mathrm{N}]$ ratio. Using this procedure, plasma cell parameters could be maintained constant for all quaternary compositions. $1 \mu \mathrm{m}$ thick lattice-matched $\operatorname{In}_{3 x} \mathrm{Ga}_{1-3 x} \mathrm{As}_{1-x} \mathrm{~N}_{x}$ epitaxial layers were grown at substrate temperatures $T_{\text {sub }}$ of 450,500 , and $550{ }^{\circ} \mathrm{C}$ and with nitrogen compositions of $0.27 \%, 0.35 \%$, and $0.55 \%$. All the samples were lightly Si doped $(n \sim 5$ 
$\times 10^{15} \mathrm{~cm}^{-3}$ ) and annealed in situ under $\mathrm{As}_{4}$ overpressure at $650^{\circ} \mathrm{C}$ for $20 \mathrm{~min}$. In situ RHEED patterns remained bright and streaky after 20 monolayers of epitaxy, indicating a smooth, layer-by-layer growth for all the samples. Lattice matching was confirmed by DXRD after growth; a single peak with less than 55 arc s full width at half maximum was observed for all samples.

Temperature-dependent photoluminescence (PL) measurements were performed with the samples mounted in a closed-cycle helium cryostat. The excitation source is a $5145 \AA \mathrm{Ar}^{+}$laser. A narrow-band Fabry-Perot etalon is used to prevent other excitation lines from reaching the sample, while a wide-band color filter is used to block the reflected $5145 \AA$ laser light from the spectrometer. The laser beam is focused to a $\sim 60 \mu \mathrm{m}$ spot, so the power density is estimated to be $500 \mathrm{~W} / \mathrm{cm}^{2}$ for an incident power of $15 \mathrm{~mW}$. The luminescence from all samples was bright enough to be measured without lock-in amplification using an Ocean Optics charge coupled device (CCD) spectrometer. Spectra from the CCD spectrometer compared favorably to those collected on a $1 \mathrm{~m}$ spectrometer using a $\mathrm{LN}_{2}$ cooled photomultiplier tube and lock-in amplification. Analysis of the decay in photoluminescence signal with increasing temperature yields the energy of shallow traps and relative trap densities.

Deep-level transient spectroscopy (DLTS) measurements were made on circular $500 \mu \mathrm{m}$ diameter gold Schottky diodes. Standard $\mathrm{Ni}-\mathrm{Ge}-\mathrm{Au}$ ohmic contacts were formed on the backside by evaporation and a subsequent anneal. Measurements were performed with the diodes placed in a variable temperature cryostat and with a SULA Technologies automated DLTS system. Capacitance-voltage measurements were performed on the diodes at room temperature to determine the background carrier concentration. DLTS measurements were made with a quiescent reverse bias of $0.5 \mathrm{~V}$ applied to the diodes, corresponding to a depletion width of $0.6 \mu \mathrm{m}$, or roughly half the film thickness. The filling pulse height was also kept constant at $0.6 \mathrm{~V}$. The DLTS signal was recorded as temperature scans for different rate windows. Minima in the $\triangle C / C$ signal correspond to thermal emission from deep levels in the bulk material. Analysis of data for several rate windows yields activation energy, capture cross section, and concentration of deep level states.

\section{RESULTS}

\section{A. Temperature-dependent photoluminescence}

All quaternary samples, grown at different substrate temperatures and with varying nitrogen content, exhibited two close but clearly distinct photoluminescent (PL) transitions, $E_{1}$ and $E_{2}$, in the intermediate temperature range $(\sim 100 \mathrm{~K})$. At low temperatures, the high-energy peak appears as a shoulder of the low-energy peak, while near room temperature, the opposite is true. As the sample temperature is increased, the intensities of both transitions are quenched. Representative data for a sample containing $0.55 \% \mathrm{~N}$ grown at $450^{\circ} \mathrm{C}$ are shown in Fig. 1. It is noted that the high-energy transition becomes dominant at higher temperatures, and quenches at much higher temperatures than the low-energy

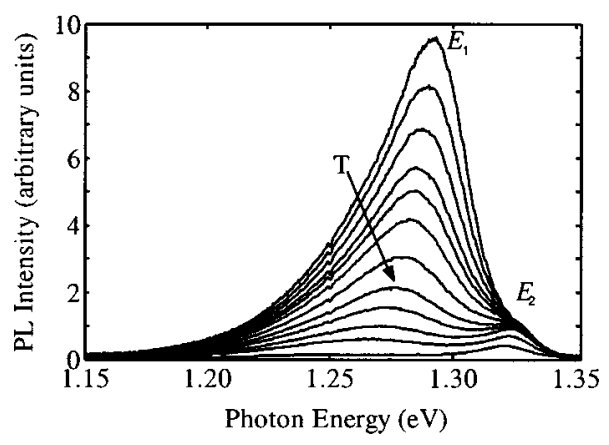

FIG. 1. Photoluminescence from a typical lattice-matched $\mathrm{In}_{0.017} \mathrm{Ga}_{0.983} \mathrm{As}_{0.9945} \mathrm{~N}_{0.0055} / \mathrm{GaAs}$ layer at various temperatures.

peak. Double-Gaussian fits were made to the PL spectra to more accurately resolve peak positions for the two transitions.

The variation of the PL peak intensity for the two transitions in a $0.55 \% \mathrm{~N}$ sample grown at $550^{\circ} \mathrm{C}$ are shown in Fig. 2(a), which illustrates the thermal quenching characteristics. The $1.326 \mathrm{eV}$ peak shows stronger thermal quenching than the $1.367 \mathrm{eV}$ peak. Similarly, Fig. 2(b) shows the temperature-dependent intensity in $0.55 \% \mathrm{~N}$ samples grown at three different substrate temperatures. It appears that the PL is quenched faster in the sample grown at higher temperature.

\section{B. Deep-level transient spectroscopy}

DLTS measurements are usually done with a Schottky diode or a $p-n$ junction. Majority carrier (electron) traps are
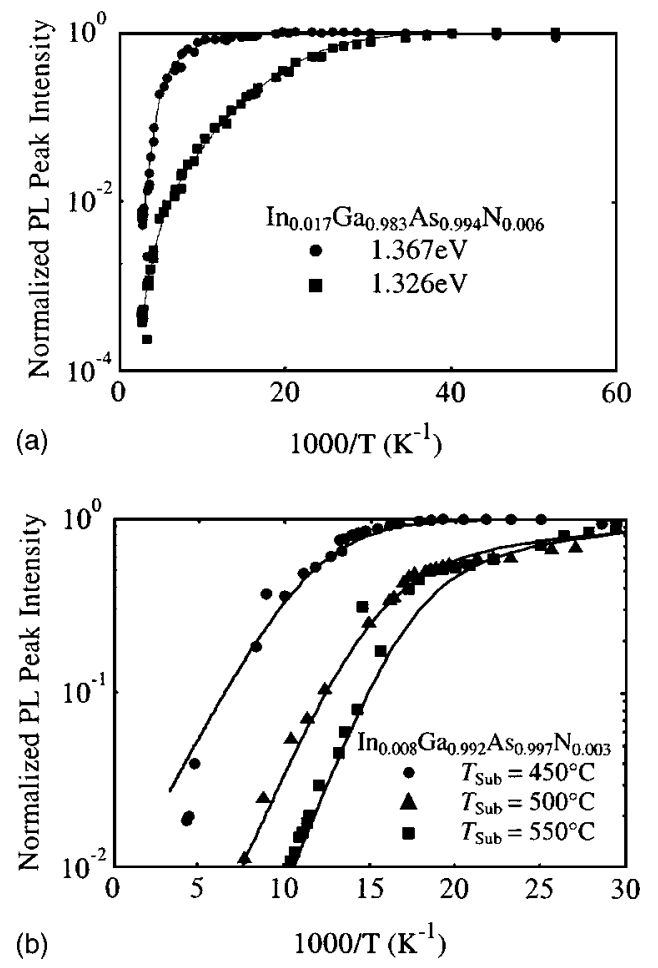

FIG. 2. Variation of photoluminescence peak intensity with inverse temperature (a) for both transitions, $E_{1}$ and $E_{2}$, in a $0.55 \% \mathrm{~N}$ alloy grown at $550^{\circ} \mathrm{C}$, and (b) for the low-energy $(13.7 \mathrm{eV})$ transition, $E_{1}$ in $0.27 \% \mathrm{~N}$ alloys grown at different substrate temperatures. 
TABLE I. Ionization energies of defects, $\Delta E_{n}$, contributing to the thermal quenching of photoluminescence in lattice-matched InGaAsN/GaAs samples with various nitrogen compositions and growth temperatures. Each $T_{\text {sub }}, \mathrm{N} \%$ combination represents a single sample. $E_{1}$ and $E_{2}$ refer to the peak energies of the transitions observed in the photoluminescence spectrum.

\begin{tabular}{|c|c|c|c|c|c|c|}
\hline \multirow{3}{*}{$\begin{array}{l}\mathrm{N} \\
\text { Content } \\
(\%)\end{array}$} & \multicolumn{6}{|c|}{ Defect ionization energy, $\Delta E_{n}(\mathrm{meV})$} \\
\hline & \multicolumn{2}{|c|}{$450^{\circ} \mathrm{C}$} & \multicolumn{2}{|c|}{$500^{\circ} \mathrm{C}$} & \multicolumn{2}{|c|}{$550^{\circ} \mathrm{C}$} \\
\hline & $E_{1}$ & $E_{2}$ & $E_{1}$ & $E_{2}$ & $E_{1}$ & $E_{2}$ \\
\hline 0.27 & 24 & 37 & 10 & 10 & 12 & 12 \\
\hline & 75 & & 45 & 45 & $\begin{array}{c}57 \\
170\end{array}$ & 51 \\
\hline 0.35 & $\begin{array}{c}18 \\
40 \\
130\end{array}$ & $\begin{array}{c}30 \\
170\end{array}$ & 26 & 25 & 26 & 16 \\
\hline 0.55 & $\begin{array}{c}13 \\
30 \\
120\end{array}$ & $\begin{array}{c}26 \\
165\end{array}$ & 29 & 15 & $\begin{array}{l}15 \\
50\end{array}$ & $\begin{array}{l}25 \\
65\end{array}$ \\
\hline
\end{tabular}

filled in an $n$-type sample with a forward bias pulse. When the diode is reverse biased, to maintain charge neutrality in the depletion region, the trapped electrons are reemitted from the deep levels with a temperature-dependent emission time constant. As the electrons escape from the traps, the depletion region width is reduced, thus causing a time-dependent change in the diode capacitance. ${ }^{15}$ The resulting capacitance transient is measured using a capacitance meter and the capacitance of the diode is sampled at times $t=t_{1}$ and $t_{2}$. The differential capacitance, $\Delta C$ is plotted for different temperatures and the function exhibits a maximum at a characteristic temperature $T_{\text {max }}\left(t_{1}, t_{2}\right)$.

The capacitance of a Schottky diode is given by

$$
C(t)=A \sqrt{\frac{q \varepsilon_{r} \varepsilon_{0}}{2}} \sqrt{\frac{N_{\mathrm{scr}}(t)}{V_{\mathrm{bi}}-V}},
$$

where $A$ is the area of the device, $N_{\text {scr }}$ is the time-dependent trapped charge density (including ionized impurities and trapped charge), $V_{\mathrm{bi}}$ is the built-in potential at the metalsemiconductor interface, $\mathrm{V}$ is the applied bias, and $q, \varepsilon_{r}$, and $\varepsilon_{0}$ have their usual meanings. The time dependence of $N_{\mathrm{scr}}(t)$ is given by $N_{\text {scr }}(t)=N_{D}-N_{\mathrm{qd}}(t)$, where $N_{D}$ is the concentration of donor atoms and $N_{q d}(t)$ is the time-dependent concentration of trapped charge in the depletion region. Rearrangement of terms yields

$$
C(t)=C_{0} \sqrt{1-\frac{N_{q d}(t)}{N_{D}}},
$$

where $N_{q d}(t)=N_{q d}(0) \exp \left(-t / \tau_{e}\right)$. The temperature dependence of the time constant, $\tau_{e}$, is given by

$$
T^{2} \tau_{e}=\frac{\exp \left(E_{T} / k T\right)}{\gamma \sigma},
$$

where $E_{T}$ is the trap activation energy. The time constant $\tau_{e \max }$, which corresponds to the temperature $T_{\max }$ described above, is obtained by differentiating $\Delta C\left(t_{1}-t_{2}\right)$ with respect to $\tau_{e}$ and setting $d \Delta C / d \tau_{e}=0$. Thus,

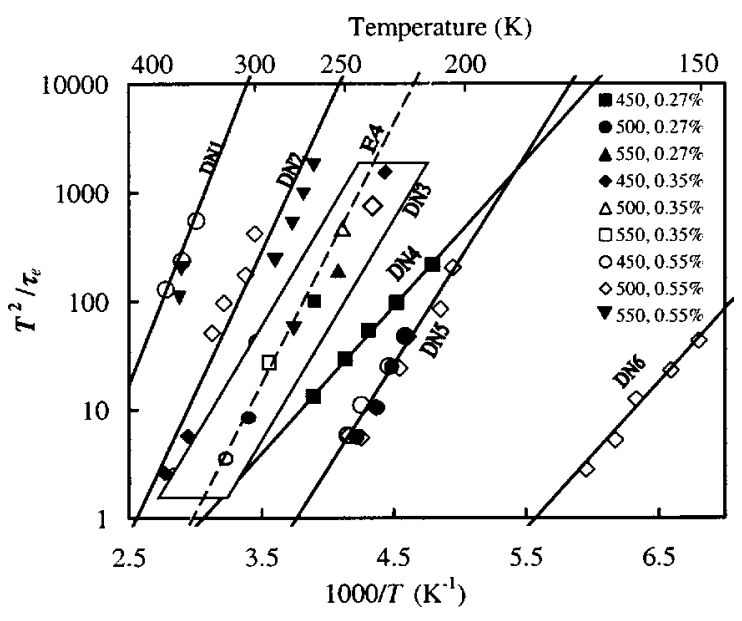

FIG. 3. Arrhenius plots of deep-level traps identified in the various latticematched InGaAsN/GaAs alloys. Solid lines represent fits to the measured data. The dashed line labeled E4 is a previously observed trap in MBE GaAs. The trapezoidal region representing trap DN3 included a reduced number of data points for clarity.

$$
\tau_{e \max }=\frac{t_{1}-t_{2}}{\ln \left(t_{2} / t_{1}\right)},
$$

$\tau_{e \text { max }}$ depends only on the sampling times. By choosing different sampling times $t_{1}$ and $t_{2}$, a series of temperature scans can be generated, from which an Arrhenius plot of $T_{\max }^{2} \tau_{e \max }$ versus $1 / T_{\max }$ is obtained.

\section{RESULTS AND DISCUSSION}

Thermal quenching of photoluminescence intensity is generally attributed to the presence of nonradiative defects and deep-level traps. In this case, the variation of intensity with temperature is given by the equation

$$
\frac{I}{I_{0}}=\frac{1}{1+c_{1} e^{-\Delta E_{1} / k T}+c_{2} e^{-\Delta E_{2} / k T}+\cdots},
$$

where for trap $n, \Delta E_{n}$ is the activation energy and $c_{n}$ is a proportionality constant related to the capture cross section,

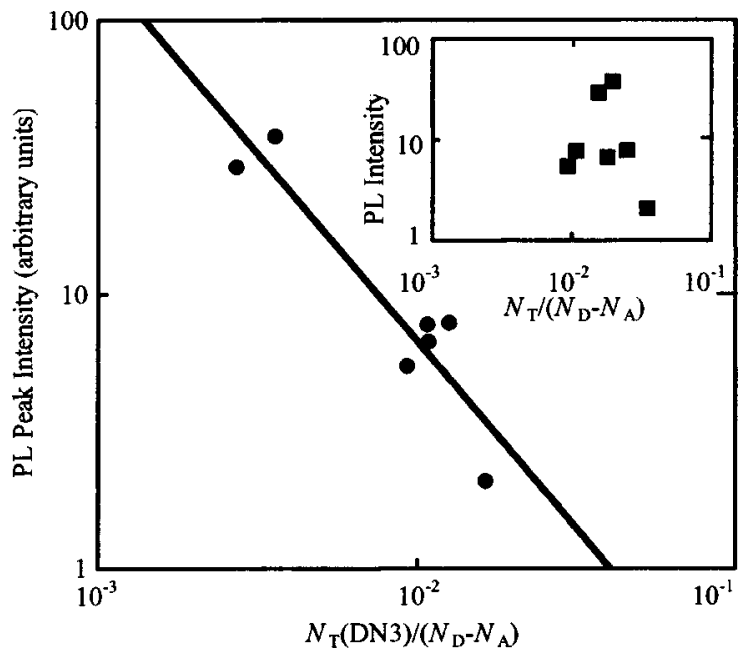

FIG. 4. Dependence of the photoluminescence intensity of lattice-matched MBE grown InGaAsN/GaAs on the density for DN3 and on the total trap density (inset). 
TABLE II. Activation energy, capture cross section, and concentration of deep-level traps identified in lattice-matched InGaAsN/GaAs epitaxial layer with various nitrogen compositions grown by molecular beam epitaxy at different substrate temperatures.

\begin{tabular}{|c|c|c|c|c|c|c|c|c|c|}
\hline \multirow{3}{*}{$\begin{array}{l}\mathrm{N} \\
\text { content } \\
(\%)\end{array}$} & \multicolumn{9}{|c|}{$T_{\text {sub }}\left({ }^{\circ} \mathrm{C}\right)$} \\
\hline & \multicolumn{3}{|c|}{450} & \multicolumn{3}{|c|}{500} & \multicolumn{3}{|c|}{550} \\
\hline & $E_{T}(\mathrm{eV})$ & $\sigma_{\infty}\left(\times 10^{-14} \mathrm{~cm}^{-2}\right)$ & $N_{T}\left(\times 10^{13} \mathrm{~cm}^{-3}\right)$ & $E_{T}(\mathrm{eV})$ & $\sigma_{\infty}\left(\times 10^{-14} \mathrm{~cm}^{-2}\right)$ & $N_{T}\left(\times 10^{13} \mathrm{~cm}^{-3}\right)$ & $E_{T}(\mathrm{eV})$ & $\sigma_{\infty}\left(\times 10^{-14} \mathrm{~cm}^{-2}\right)$ & $N_{T}\left(\times 10^{13} \mathrm{~cm}^{-3}\right)$ \\
\hline \multirow[t]{2}{*}{0.27} & 0.27 & $6.0 \times 10^{-3}$ & 1.0 & 0.45 & 1.5 & 3.4 & 0.45 & 1.5 & 3.6 \\
\hline & 0.27 & 10.0 & 0.4 & 0.39 & 8.0 & 3.7 & 0.43 & 1.5 & 3.2 \\
\hline \multirow[t]{3}{*}{0.35} & 0.45 & 1.5 & 2.8 & 0.45 & 1.5 & 5.4 & 0.62 & 1.5 & 1.1 \\
\hline & & & & & & & 0.45 & 1.5 & 18.1 \\
\hline & & & & & & & 0.27 & 10.0 & 11.5 \\
\hline \multirow[t]{4}{*}{0.55} & 0.62 & 1.5 & 5.4 & 0.52 & 2.0 & 15.8 & 0.62 & 1.5 & 16.2 \\
\hline & 0.45 & 1.5 & 13.6 & 0.45 & 1.5 & 6.8 & 0.52 & 2.0 & 11.3 \\
\hline & 0.39 & 8.0 & 3.6 & 0.43 & 1.5 & 11.3 & 0.45 & 1.5 & 9.7 \\
\hline & & & & 0.27 & 10.0 & 5.4 & 0.43 & 1.5 & 15.7 \\
\hline
\end{tabular}

trap density, and electron-hole wave function overlap. Data similar to that shown in Fig. 2 have been obtained for all samples and analyzed according to Eq. (5). The dashed lines in Fig. 2(a) indicate fits to the measured data. The results are outlined in Table I. It is apparent that PL quenching at high temperatures is caused by a number of shallow and nonshallow (nonhydrogenic) defect states which may be related to impurities, native defects such as vacancies, or complex defects. Though lower growth temperatures have shown to degrade luminescence efficiency, ${ }^{5}$ the samples grown at lower temperatures in the present study show the strongest luminescence. We attribute this improvement to reduced incorporation of nitrogen-related defects at low growth temperatures and their removal during the subsequent in situ anneal.

Figure 3 depicts the Arrhenius plots of the deep-level traps identified in the various alloys grown at different substrate temperatures. Table II lists the measured trap characteristics in all the samples and Table III describes the trends of their occurrences in the samples. A few dominant features are evident. The trap labeled DN3 is present in all the samples, irrespective of alloy composition and growth temperature. It is therefore a dominant trap in this quaternary alloy system, which may be related to impurities present in nitrogen. It is also very similar to the traps labeled E3 and E4 in MBE GaAs. ${ }^{6}$ In contrast, traps labeled DN4 and DN6 occur in one sample each. Their origin is therefore attributed to unknown impurities. In general, trap densities increase with $\mathrm{N}$ content in the alloy and growth temperature. Therefore, nitrogen itself or an impurity associated with the nitrogen source is probably responsible for the formation and presence of most defects that behave as deep-level traps in these alloys.

We have investigated the relationship between PL intensity and trap density in the various samples. In doing so, we have plotted the total trap density and the density of the DNB center, normalized to the measured background carrier concentration, versus the peak PL intensity, as seen in Fig. 4. While we find no correlation between the total trap density and PL peak intensity (inset), it is evident that there is an inverse relationship between the DN3 trap concentration and PL intensity. Since the trap concentration also increases with nitrogen content in the alloys, it is quite likely that DN3 is related to an impurity in the nitrogen source, which reduces

TABLE III. Characteristics and trends in the occurrence of deep-level traps in lattice-matched InGaAsN/GaAs epitaxial layers grown by molecular beam epitaxy.

\begin{tabular}{|c|c|c|c|c|}
\hline Traps & $E_{T}(\mathrm{eV})$ & $\sigma_{\infty}\left(\times 10^{-16} \mathrm{~cm}^{-2}\right)$ & $N_{T}\left(\times 10^{13} \mathrm{~cm}^{-3}\right)$ & Comments \\
\hline DN1 & 0.62 & 1.5 & $1.0-16.0$ & $\begin{array}{l}\text { Present only in samples with } 0.55 \% \mathrm{~N} \text {; trap density } \\
\text { increases with growth temperature. Also observed in } \\
\text { GaAsN(Ref. 19). }\end{array}$ \\
\hline DN2 & 0.52 & $2.0 \times 10^{2}$ & $10.0-15.0$ & $\begin{array}{l}\text { Present in samples with } 0.35 \% \text { and } 0.55 \% \mathrm{~N} \text {; trap } \\
\text { density increases with } \mathrm{N} \text { content and growth } \\
\text { temperature; Also observed in unstrained InGaAs } \\
\text { (Ref. 20). }\end{array}$ \\
\hline DN3 & 0.44 & $1.5 \times 10^{2}$ & $0.5-20.0$ & $\begin{array}{l}\text { Present in all samples. Density generally increases } \\
\text { with } \mathrm{N} \text { content and growth temperature; Similar to } \\
\text { traps E3 and E4 in MBE GaAs (Ref. 16). }\end{array}$ \\
\hline DN4 & 0.27 & $6.0 \times 10^{-1}$ & 1.0 & $\begin{array}{l}\text { Present only in sample with } 0.27 \% \mathrm{~N} \text { grown at } \\
450^{\circ} \mathrm{C} \text {. }\end{array}$ \\
\hline DN5 & 0.39 & $8.0 \times 10^{2}$ & $0.2-3.7$ & Occurs sporadically. \\
\hline DN6 & 0.27 & $1.0 \times 10^{3}$ & 0.4 & $\begin{array}{l}\text { Present only in one sample with } 0.55 \% \text { Ngrown at } \\
\qquad 500^{\circ} \mathrm{C} \text {. }\end{array}$ \\
\hline
\end{tabular}


luminescence efficiency. Impurities in the nitrogen--argon gas mixture are most likely $\mathrm{O}_{2}$ and $\mathrm{H}_{2} \mathrm{O}$. Concentrations are $\leqslant 1$ ppm for both. In situ measurements of the gas using the residual gas analyzer on the growth chamber show no signs of oxygen $(<100 \mathrm{ppb})$, but are inconclusive for $\mathrm{H}_{2} \mathrm{O}$ due to the proximity of strong $\mathrm{N}$ peaks. An upper limit on $\mathrm{H}_{2} \mathrm{O}$ concentration is approximately $500 \mathrm{ppb}$. For nitrogen concentrations near $1 \times 10^{20} \mathrm{~cm}^{-3}(0.45$ at. \%) from a $15 \% \mathrm{~N}_{2} / \mathrm{Ar}$, a $500 \mathrm{ppb} \mathrm{H}_{2} \mathrm{O}$ impurity could result in a defect concentration of approximately $1.5 \times 10^{14} \mathrm{~cm}^{-3}$ for $\mathrm{O}$, which agrees with observed trap concentrations.

The measured capture cross sections of all the traps, except DN4 is large $\left(>10^{-15} \mathrm{~cm}^{-2}\right)$, which is generally associated with attractive centers. The capture cross section of DN4 is within the range associated with neutral centers.

\section{CONCLUSIONS}

We have studied photoluminescence and deep levels in molecular beam epitaxial $\mathrm{In}_{y} \mathrm{Ga}_{1-y} \mathrm{As}_{1-x} \mathrm{~N}_{x}$ alloys latticematched to GaAs. A comparison of the two measurements shows a correlation between the dominant DN3 deep level trap with an activation energy of $0.44 \mathrm{eV}$ and observed luminescent intensity of the samples. This deep level is similar in characteristics to the E3 and E4 states previously observed in MBE GaAs. ${ }^{16}$ Since this trap is present in all samples and increases with nitrogen content, we attribute it to nitrogenrelated impurities in the source gas mixture. The nearby E3 trap has been convincingly linked to oxygen-related defects, ${ }^{17,18}$ but no link has yet been shown for the E4 state. It is worth noting, however, that the energy separation between these two states is only $0.06 \mathrm{eV}$, and that the slope (energy) of the DN3 state matches E3 better than E4. This small energy difference from a well-established oxygenrelated deep level, along with an order-of-magnitude agreement between known gas impurity concentrations and trap densities, leads us to speculate that $\mathrm{O}_{2}$ or $\mathrm{H}_{2} \mathrm{O}$ source gas impurities may be responsible for the observed DN3 trap.

\section{ACKNOWLEDGMENT}

The work was supported by the Air Force Office of Scientific Research (DoD MURI program) under Grant No. F49620-00-1-0328.

${ }^{1}$ J. F. Geisz and D. J. Friedman, Semicond. Sci. Technol. 17, 769 (2002). ${ }^{2}$ M. H. Ya, W. Z. Cheng, Y. F. Chen, and T. Y. Lin, Appl. Phys. Lett. 81, 3386 (2002).

${ }^{3}$ C. H. Fischer, P. Bhattacharya, and P. C. Yu, Electron. Lett. 39, 1537 (2003).

${ }^{4}$ U. Tisch, E. Finkman, and J. Salzman Appl. Phys. Lett. 81, 463 (2002).

${ }^{5}$ A. R. Kovsh, J. S. Wang, L. Wei, R. S. Shiao, J. Y. Chi, B. V. Volovik, A.

F. Tsatsul'nikov, and V. M. Ustinov J. Vac. Sci. Technol. B 20, 1158 (2002).

${ }^{6}$ G. Mussler, L. Daweritz, and K. H. Ploog, Appl. Phys. Lett. 83, 1343 (2003).

${ }^{7}$ Y. Fedorenko, T. Jouhti, E. M. Pavelescu, S. Karirinne, J. Kontinnen, and M. Pessa, Thin Solid Films 440, 195 (2003).

${ }^{8}$ M. C. Larson, M. Kondow, T. Kitatani, K. Nakahara, K. Tamura, H. Inoue, and K. Uomi, IEEE Photonics Technol. Lett. 10, 188 (1998).

${ }^{9}$ M. Fischer, D. Gollub, M. Reinhardt, M. Kamp, and A. Forchel, J. Cryst. Growth 251, 353 (2003).

${ }^{10}$ V. A. Odnoblyudov, A. Y. Egorov, A. R. Kovsh, V. V. Mamutin, E. V. Nikitina, Y. M. Shernyakov, M. V. Maksimov, and V. M. Ustinov, Tech. Phys. Lett. 29, 433 (2003).

${ }^{11}$ M. A. Wistey, S. R. Bank, H. B. Yuen, L. L. Goddard, and J. S. Harris, Electron. Lett. 39, 1822 (2003).

${ }^{12}$ D. A. Louderback, M. A. Fish, J. F. Klein, D. K. Serkland, K. D. Choquette, G. W. Pickrell, R. V. Stone, and P. S. Guilfoyle, IEEE Photonics Technol. Lett. 16, 963 (2004).

${ }^{13}$ D. Gollub, S. Moses, A. Fischer, M. Kamp, and A. Forchel, Electron. Lett. 40, 427 (2004).

${ }^{14}$ Q. Gao, H. H. Tan, C. Jagadish, B. Q. Sun, M. Gal, L. Ouyang, and J. Zou, Appl. Phys. Lett. 84, 2536 (2004).

${ }^{15}$ D. V. Lang, J. Appl. Phys. 45, 3023 (1974)

${ }^{16}$ G. M. Martin, A. Mitonneau, and A. Mircea, Electron. Lett. 13, 191 (1977).

${ }^{17}$ H. C. Alt, Semicond. Sci. Technol. 6, B121 (1991).

${ }^{18}$ A. Balcioglu, R. K. Ahrenkiel, and D. J. Friedman J. Appl. Phys. 93, 3635 (2003).

${ }^{19}$ P. Krispin, S. C. Spruytte, J. S. Harris, and K. H. Ploog. Physica B 308310, 870 (2001)

${ }^{20}$ D. Pal, E. Gombia, R. Mosca, A. Bosacchi, and S. Franchi, J. Appl. Phys. 84, 2965 (1998) 